\title{
La Pologne et les Polonais dans l'œuvre de Georges Simenon : vers un orientalisme intra-européen
}

\section{Poland and the Poles in the Works of Georges Simenon: towards an Intra-European Orientalism}

PrzemysŁaw Szczur [przemyslaw.szczur@up.krakow.pl]

Uniwersytet Pedagogiczny im. Komisji Edukacji Narodowej w Krakowie, Pologne

\section{RÉSUMÉ}

Cet article a pour objectif d'examiner la représentation de la Pologne et des Polonais dans l’ouvre de Georges Simenon, dans une perspective inspirée principalement de l'imagologie et de la théorie de l'orientalisme. Sont pris en compte aussi bien des reportages que des romans et des nouvelles de l'auteur belge. Lanalyse des textes aboutit à l'identification d'un certain nombre de traits caractéristiques de la vision simenonienne de la Pologne et des Polonais, tels que : négativisation, exotisation, racialisation, érotisation, déshumanisation, animalisation, altérisation et étrangéisation linguistique. Rien que cette liste suggère la tonalité négative qui domine dans le portrait textuel du pays et de ses habitants. Quelques notes plus positives sont également signalées, mais l'accumulation de traits négatifs est surtout interprétée comme une forme d’orientalisme intra-européen, autrement dit un discours idéologiquement chargé dans le cadre duquel la Pologne, et plus largement, l'Europe centrale et orientale, sont opposées à un Ouest européen incarnant la norme civilisationnelle et humaine. La théorie de l'orientalisme s'avère ainsi un outil pouvant servir non seulement à la description de la domination symbolique de l'Europe occidentale colonisatrice sur les pays colonisés, mais aussi sur l'Orient intra-européen.

\section{MoTS CLÉS}

Georges Simenon ; Pologne ; Polonais ; Imagologie ; Orientalisme

\begin{abstract}
The aim of this paper is to examine the representation of Poland and the Poles in the works of Georges Simenon, from a perspective inspired mainly by imagology and the theory of orientalism. This includes Simenon's reportages as well as novels and short stories. The analysis of the texts leads to the identification of a certain number of characteristic features of the Simenonian vision of Poland and the Poles, such as: negativization, exoticization, racialization, eroticization, dehumanization, animalisation, otherization and linguistic strangerization. This list alone suggests the negative tone that dominates in the textual portrait of the country and its inhabitants. Some more positive notes are also reported, but the accumulation of negative traits is mainly interpreted as a form of intra-European orientalism, that is an ideologically charged discourse in the context of which Poland, and more broadly, Central and Eastern Europe, are opposed to a European West embodying the civilizational and human standard. The theory of orientalism thus turns out to be a tool
\end{abstract}


which can be used not only to describe the symbolic domination of colonizing Western Europe over the colonized countries, but also over the intra-European East.

\author{
KEYWORDS \\ Georges Simenon; Poland; Poles; Imagology; Orientalism
}

REÇU 2020-06-29; ACCEPTE 2020-09-08

Lauteur de cette étude bénéficie d'un financement octroyé par le Centre national de la recherche scientifique polonais (National Science Centre, Poland, research project 2018/30/M/HS3/00153).

\title{
1. Introduction
}

Pierre Assouline, l'un des biographes de Georges Simenon, caractérise ainsi la vision simenonienne des étrangers :

La racine de sa vision de létranger se trouve dans un moment de la vie de Simenon : quand il avait 15 ans, sa mère a ouvert une pension de famille chez eux, qui logeait cinq ou six étudiants étrangers d'Europe de l'Est. Parfois Juifs, très désargentés, très pauvres, ils étaient souvent étudiants en médecine. Il les a écoutés à table, c'est de là qu'il connaît bien le roman russe, notamment Dostoïevski, il a beaucoup appris en les écoutant. Sa vision de l'étranger est très intéressante. Il a une vraie compassion, contrairement à ce que l’on pourrait croire (Assouline 2017).

Stanley Eskin, un autre biographe de l'écrivain belge, a, pour sa part, constaté : "Lattitude de Simenon à leur égard varie beaucoup, allant de la dérision jusquà la compassion et presque l'idéalisation en passant par le dédain et la sympathie condescendante (Eskin 1990 : 45). Ainsi la question de l'« alterophilie » ou de l'« alterophobie » de l'écrivain belge fait-elle débat. Sans prendre position dans ce « conflit des interprétations » concernant l'attitude de l'homme-Simenon envers les étrangers, je souhaiterais ici me demander ce qui en est dans l'œuvre du reporter, nouvelliste et romancier. Pour ce faire, je m’intéresserai à un groupe particulier d'étrangers, particulièrement nombreux en Belgique et en France dans l'entre-deux-guerres, à savoir les Polonais. J'analyserai leur image et celle de leur pays dans les reportages Europe 33 et Peuples qui ont faim, publiés en France, en 1933 et 1934, respectivement, dans l'hebdomadaire Voilà et le quotidien Le Jour, mais également dans l'œuvre fictionnelle : les nouvelles Waldemar Strvzeski (1930) et Stan le Tueur (1938) ainsi que les romans Pietr le Letton (1931), Le Fou de Bergerac (1932), L'Outlaw (1941) et Maigret et son mort (1948).

Dans les rares textes qui ont jusqu’à présent effleuré ce sujet, sans vraiment l'explorer, c'est la perspective biographico-génétique, que représente aussi Assouline, qui tient beaucoup de place. À titre d'exemple, Agnieszka Pantkowska s'est penchée sur les racines de l'image simenonienne de la Pologne, pour noter que l'ouvre de Simenon-reporter avait servi de base à celles du ro- 
mancier et nouvelliste, comme on le voit dans cet extrait : "Les impressions acquises lors de ses voyages en Pologne ont certainement inspiré la nouvelle Waldemar Strvzeski (Pantkowska 2006: 128). Concernant ce texte particulier, un simple examen de la chronologie permet de démentir cette interprétation génétique de la chercheuse : la nouvelle a été écrite en 1929-1930 (Waldemar Strvzeski s.d.), sa création précède donc le voyage de Simenon en Pologne, effectué en 1933 (Lemoine, Carly 2003 : 80). Il en est de même de deux autres œuvres où apparaissent des personnages de Polonais : le premier Maigret, Pietr le Letton (Pietr le Letton s. d.), rédigé à peu près à la même époque que la nouvelle, et Le Fou de Bergerac (Le Fou de Bergerac s. d.), texte légèrement postérieur, mais toujours antérieur au voyage polonais de lécrivain. Si on relève bien des motifs communs aux reportages, romans et nouvelles, il est souvent impossible, pour des raisons purement chronologiques, de considérer l'œuvre du romancier et nouvelliste comme une transposition des expériences du reporter. Tout en signalant quelques convergences entre les pans référentiel et fictionnel de l'œuvre simenonienne, l'analyse qui suivra n'aura donc pas pour objectif d'établir des relations de filiation entre les textes.

En effet, pour les raisons susmentionnées, l’on ne peut pas dire qu'après avoir fait l'expérience de la réalité polonaise, Simenon en a simplement proposé un reflet dans ses textes fictionnels. Il semble bien que ce soit l'imagination du romancier et nouvelliste qui ait ouvert la voie à l'œuvre du reporter, et non l'inverse. Au lieu de m’atteler à une recherche génétique, je vais donc me situer plutôt dans la lignée de l'imagologie littéraire, c'est-à-dire de « l'étude des images de l'étranger dans une œuvre, une littérature " (Pageaux 2007 : 27). Comme on le sait, dans l'une de ses acceptions de base, le terme d'image désigne " un schème imaginaire [...] qui détermine les façons de percevoir autrui »(Bordas 2002 : 289). À mon sens, cette définition présuppose que l’on se concentre, dans une analyse de type imagologique, sur les éléments récurrents - qui permettent de reconstituer ce " schème " - et le facteur subjectif - l' "imaginaire " - propre à l'auteur étudié. Cette idée générale me guidera tout au long de mes analyses. Citer des fragments de textes simenoniens me permettra d'identifier les « constellations verbales» ou encore «l'arsenal notionnel » ayant servi à créer l'image de la Pologne et des Polonais, car c'est l'un des constituants fondamentaux de toute image, selon Daniel-Henri Pageaux (1995 : 142). J'essaierai aussi d'en dégager la logique interne grâce à laquelle de ces " constellations verbales " émerge une image relativement cohérente. C’est ainsi qu’apparaîtra la dimension subjective susmentionnée, mais au lieu de la rattacher à une analyse de la psychologique individuelle de l'homme-Simenon (ce qui relèverait d'une sorte de psychanalyse littéraire posthume), je la relierai à l'idéologie qui me semble sous-tendre ses textes, ainsi qu'aux spécificités de la pratique simenonienne des genres dans lesquels l'écrivain dessine sa Pologne et ses Polonais, à savoir, principalement, le reportage et le roman.

Mon insistance sur la dimension idéologique des œuvres analysées, déjà suggérée par le titre de cet article, constituera la mise en application de l'un des principes méthodologiques de base de toute recherche imagologique, ainsi formulé par Daniel-Henri Pageaux : « Lorsque nous interrogeons des textes littéraires intéressant l'imagologie, demandons-nous [...] dans quelle mesure cette représentation de l'étranger peut être tributaire d'une certaine option idéologique " (Pageaux 2007 : 35). C'est au prix d'un tel questionnement qu'il me sera possible de détacher l'image simenonienne de la Pologne et des Polonais des aléas de la psychologie individuelle et d'y repérer une composante collective. Observer comment sont représentés un pays d'Europe de l'Est, 
ses habitants et sa culture me permettra ainsi d’ajouter un chapitre intra-européen à l'histoire de l'orientalisme occidental dont l'écrivain belge fut sans doute, vu son immense popularité, l'un des adeptes les plus influents.

\section{Négativisation et exotisation}

Selon une conception courante du reportage, de type mimétique, ce genre offrirait une sorte de reflet linguistique direct de la réalité. La définition du mot que propose Le Trésor de la langue française informatisé va justement dans ce sens ; le reportage y est défini comme "Action de recueillir à leur source des informations d'actualité ou d'intérêt documentaire et de les relater directement, aussi objectivement que possible» (Reportage s. d.). Pourtant, les spécialistes du genre ont depuis longtemps relevé les limites de cette prétendue « objectivité ». Bernard Alavoine, qui a travaillé sur les reportages de Simenon, souligne ainsi que : « [...] le reportage se distingue [...] de la relation de voyage par sa situation éditoriale et sa thématique : c'est à la fois l'actualité et l'attrait pour le sensationnel qui vont prévaloir sur les aventures du narrateur ou l'histoire du pays visité » (Alavoine 2001a : 176). La mimésis est donc en loccurrence relativisée par des contraintes génériques, et notamment le penchant du genre pour le sensationnel. Limage simenonienne de la Pologne penche en effet de ce côté-là. Voici deux fragments de la série de reportages Europe 33 que l'on peut interpréter dans ce sens :

Les routes ne sont pas des routes! Les gares sont des baraques! Les maisons sont délabrées comme les villes, les hôpitaux à peu près inexistants ! Il n'y a pas d'écoles, pas d'universités, pas d’armée polonaise ! Il n'y a rien ! [...]

Et les bicoques puantes, les paysans illettrés, les moyens de transport rarissimes, les œuvres sociales inconnues! (Simenon $2001: 780$ ).

Le sensationnalisme se manifeste ici notamment par la mise en valeur des émotions du sujet parlant que signalent les points d'exclamation ; or, est « sensationnel », ce qui " produit une forte impression, un effet de surprise sur le public » (Sensationnel s. d.). En mettant en avant l'émoi que provoque chez lui la réalité polonaise, le reporter semble chercher à le communiquer - au sens non seulement d'en parler, mais aussi de le faire partager - à ses lecteurs et lectrices. Au niveau des figures, c'est l'hyperbolisation qui est susceptible de produire un fort effet sur ces derniers. En l'occurrence, de la multiplication de tournures négatives, l’on passe à une véritable néantisation de la Pologne qui relève clairement de l'hyperbole. Laccumulation de négations partielles aboutit à une négation totale. Dans les extraits cités, l'on a donc affaire à la fois au respect d'une contrainte générique sensationnaliste et à une forte présence de la subjectivité du reporter. Ce qui y frappe aussi, c’est l'omniprésence de la négativité : elle se manifeste non seulement par la prolifération de négations, déjà relevée, mais également par la multiplication d'adjectifs aux préfixes privatifs et/ou aux connotations péjoratives. Daniel-Henri Pageaux a recommandé à tout imagologue d' " être attentif à l'adjectivation qui permet certains procédés de qualification » (Pageaux 1995 : 142). Or, dans ce cas, il s'agit d'une adjectivation et qualification exclusivement négative. 
Au-delà des aspects linguistiques signalés, cette négativisation simenonienne de la Pologne s’effectue, au niveau du contenu représentationnel des reportages, par le biais d’un misérabilisme très poussé. Ainsi, dans sa description de Vilna, dans Europe 33, Simenon-reporter note-t-il : « Il $\mathrm{y}$ a surtout de grandes maisons tristes où l'on remplace les vitres par du carton à mesure qu'elles cassent, des rues neigeuses où filent des traîneaux puants, et des pauvres, des pauvres tous les dix mètres, des pauvres plus pauvres que dans n'importe quel pays du monde " (Simenon 2001 : 775). Dans ce paysage urbain dysphorique, l’on retrouve l'adjectivation négativisante déjà relevée. La Pologne se trouve ici érigée en une sorte de mètre de Sèvres de la misère. Lorsque Simenon raconte sa visite de Vilna, dans le reportage Peuples qui ont faim, en 1934, les images sont tout aussi misérabilistes. Il qualifie les maisons de «bicoques infectes» (Simenon 2001 : 859). En plein hiver, il portraiture "des femmes et des enfants pieds nus" (Simenon $2001: 859$ ). Il cite aussi les propos de l'homme qui lui sert de guide et qui prétend que ceux qu'ils voient « ne se déshabillent pas de tout l'hiver» (Simenon $2001: 859$ ), ce qui devient prétexte à l'évocation de leur odeur. Dans une famille à qui ils rendent visite, on vit à douze dans une seule pièce et les enfants sont " en haillons " (Simenon 2001 : 860). Dans une épicerie qui fait aussi office de cabaret et où ils prennent leur repas, les seules denrées qu'ils trouvent sont des maquereaux en boîte, du pain noir et de la vodka. La misère, quelle soit matérielle ou humaine, est ici mise en récit et en spectacle. Tel un visiteur dans un musée du dénuement, Simenon raconte ses pérégrinations à travers une ville polonaise miséreuse. Son guide exhibe devant lui des Polonais misérables tels des spécimens dans un zoo humain. Sous la plume de lécrivain belge, la misère polonaise devient proprement « spectaculaire », participant de la mise en place d'une certaine forme d'exotisme à la fois national et social, un bourgeois belge, envoyé d'un journal français, contemplant le spectacle de la pauvreté des marginaux polonais ${ }^{1}$. Fort d'une double distance : de nationalité et de classe, Simenon-reporter pose sur les choses et les êtres un regard dénué de toute empathie, curieux et dégoûté en même temps.

Le récit de la visite dans un asile de nuit de Varsovie fait appel au même paradigme descriptif misérabiliste. C'est partout la boue, les fenêtres sont « aveuglées par des volets » (Simenon 2001 : 861), lodeur est infecte, de vieux hommes dorment debout ou par terre, la lumière est « mauvaise », les pieds des hommes «tuméfiés » (Simenon 2001 : 862). Et Simenon de résumer : « [...] il y a ici des échantillons de toutes les misères physiques, de toutes les déformations qui peuvent advenir à un corps d'homme» (Simenon 2001 : 862). L'on pourrait être tenté de voir dans ce portrait misérabiliste de la Pologne une peinture réaliste des conséquences de la crise économique des années 1930 parmi les classes défavorisées. Ce qui est étonnant, c'est que cette accumulation d'images du dénuement le plus total se poursuive, dans une version atténuée, certes, dans l'évocation des classes privilégiées polonaises et de leur cadre de vie. Pour elles, selon Simenon, « on a bâti [...] des immeubles neufs » mais les « entourent encore des terrains vagues où l'on se crotte jusqu'aux genoux » et, à l'intérieur de ces immeubles, la cuisine "sert pour vingt ou trente ménages " (Simenon 2001 : 863). Dans la noblesse, « Les hôtels particuliers sont délabrés, et y errent comme des ombres des gens qui cherchent à vendre leurs bibelots » (Simenon 2001 : 863). La misère se transforme ainsi en une caractéristique nationale des Polonais, toutes classes sociales confondues. Cette misère «polonaise " permet à Simenon d'expliquer l'émigration de nombreux

1 Comme le rappelle Jean-Marc Moura, l'une des définitions de l'exotisme, est la « réduction de létranger au spectaculaire » (Moura $1997: 13$ ). 
habitants du pays vers la Belgique et la France. Pourtant, à l'en croire, cette émigration ne change pas grand-chose à la situation de ceux qui la choisissent : les émigrants semblent emporter la misère dans leurs bagages et, dans les portraits qu’il dresse de ceux qui se sont installés à Paris ou dans le Nord, la pauvreté extrême revient toujours tel un leitmotiv.

La misère est donc aussi le signe distinctif des immigrés polonais en France portraiturés par Simenon dans ses romans et nouvelles. Lécrivain s'intéresse alors surtout à sa dimension criminogène : les vocations criminelles qu'il remarque parmi les migrants apparaissent comme résultat du dénuement extrême dans lequel ils sont plongés. C'est ainsi que les Polonais se retrouvent le plus souvent dans les intrigues policières simenoniennes dans le rôle du criminel. Ces histoires criminelles dont ils sont les héros constituent un autre élément clé de leur image, une composante que l'on pourrait nommer, à la suite de Daniel-Henri Pageaux, « le scénario » (Pageaux 1995 : 142); il ne s'agit plus de qualités (ou plutôt défauts) qui leur sont prêtées mais de leur fonction diégétique. À titre d’exemple, la misère "polonaise » criminogène devient génératrice de l'intrigue romanesque dans L'Outlaw. La première partie du roman retrace principalement les pérégrinations parisiennes d'un marginal d’origine polonaise, Stanislas Sadlak, taraudé par la faim. Le héros tente par tous les moyens, y compris criminels, de se procurer de l'argent, simplement pour avoir de quoi manger. Ce périple d'un crève-la-faim, à la fois tragique et burlesque, tant ce raté simenonien typique y est maladroit et pitoyable, répond en écho à une notation du reportage Peuples qui ont faim où Simenon remarquait : « [...] comme je m’approchais de l'est, j’ai rencontré la Faim » (Simenon 2001 : 855). La majuscule signale ici l'aspect extrême du phénomène et la nécessité de renforcer le sens habituel du mot « faim » pour décrire une réalité est-européenne. Dans L'Outlaw, Stanislas Sadlak transporte cette réalité en France. Le roman contient aussi l'évocation d'une famille polonaise habitant la Zone de Saint-Ouen et se servant de ses enfants pour mendier. Les Polonais simenoniens restent ainsi figés dans une altérité non seulement nationale, mais aussi sociale.

Selon Daniel-Henri Pageaux, l'analyse imagologique devrait, entre autres, s'intéresser « à tout processus de comparaison qui permet de [...] comprendre comment peuvent sécrire des processus d'appropriation de l'étranger [...] ou d'éloignement, d'exotisation » (Pageaux 1995 : 142). Une série de citations nous permettra de relever les comparaisons dont se sert Simenon pour construire sa Pologne textuelle :

[...] j’ai parcouru la campagne. Mais le mot correspond-il à ce que nous connaissons ? Nos fermes sont des palais, nos paysans des grands seigneurs [...] La plus mauvaise route de France est une piste idéale auprès des routes de Pologne. Au surplus, ce ne sont pas des routes. Ce sont des chemins. Ce sont des ornières. [...] Les maisons ? J’ai rencontré les mêmes au cœur de l'Afrique... (Simenon $2001: 778$ )

En dehors de quelques grands hôtels, de quelques rues, de quelques familles, le Français est aussi dépaysé qu’en Chine... (Simenon 2001 : 783)

J'ai pénétré dans les huttes des Pygmées, au Congo. J’ai visité les égouts de Paris. Cette odeur-ci est plus terrible [...]. Je suis dans un asile de nuit polonais (Simenon $2001: 861$ ).

Les comparaisons qui apparaissent dans ces extraits éloignent la Pologne de la France et la rapprochent de l'Afrique et de la Chine. Un pays européen se trouve explicitement "africanisé » et « sinisé ». Aucune « appropriation » de la réalité polonaise par Simenon ne semble possible; au 
contraire, il se livre à une entreprise d'éloignement et d'exotisation radicale. Il s'agit d'un éloignement hyperbolique : la distance géographique relativement modeste entre la France (ou la Belgique) et la Pologne se trouve démultipliée au niveau imaginaire, et le pays entier, imaginairement transporté en Afrique et en Asie, ces contrées exotiques par excellence pour le public européen. On voit ainsi se vérifier une constatation de Pierre Halen sur l'exotique comme un « lointain » qui, au sens strict, peut ne pas en être un ; en effet, selon le chercheur, « [...] la distance supposée par la notion de 'lointain' peut [...] n’avoir aucune signification en termes d'espace " (Halen 1997 : 202). L'exemple polonais prouve que rien n'empêche l'exotisation d'un pays européen assez proche, à condition que la distance géographique réelle soit démultipliée par la façon de le représenter. La distanciation est aussi instaurée par l'insistance sur le dépaysement que le Français est censé immanquablement ressentir en visitant la Pologne. Ce dépaysement est l'effet d'une frontière ontologique quasiment infranchissable que Simenon érige entre la Pologne et la France. L'image du pays est construite par le biais d'un raisonnement différentialiste. Cette différence ontologique radicale qu'incarne la Pologne se manifeste dans une quasi-défaillance de la langue française devant les réalités polonaises. Les mots eux-mêmes perdent tout pouvoir de nomination face à des référents proprement innommables. Faute de mieux, une chaîne sémantique métonymique se met en place : les routes deviennent des chemins, ceux-ci se transforment en ornières. Ces hésitations sémantiques ne font que traduire une différence radicale entre les référents polonais et ceux français qui apparaissent comme les seuls dont la langue française puisse adéquatement rendre compte.

Les procédés examinés ci-dessus, qui relèvent tous d'une certaine forme d'exotisation, me semblent dessiner assez clairement l'horizon idéologique qui détermine l'image de la Pologne dans l'œuvre simenonienne : c'est celui de l'orientalisme, ce complexe idéologique massif qui a conditionné la représentation symbolique des divers ailleurs de l'Europe occidentale tout au long du XIX', et encore au XX ${ }^{\mathrm{e}}$ siècle. L’une des définitions de l'orientalisme proposées par Edward Said en fait un "style de pensée fondé sur la distinction ontologique et épistémologique entre l'Orient' et [...] l'Occident' " (Said 2005 : 15). Or, comme nous venons de le voir, c'est justement un style de raisonnement dichotomique qui se trouve au fondement de l'image simenonienne de la Pologne. Cette dernière est opposée à la France. Autre dimension orientaliste de cette image : dans la mesure où la Chine et l'Afrique figurent parmi les objets traditionnels du discours orientaliste, l'africanisation et la sinisation de la Pologne sont synonymes de son orientalisation. Exotisée (l'orientalisme est une forme d'exotisme) par sa transformation en une sorte de Chine ou d'Afrique intra-européenne, la Pologne devient, avec les autres pays de l'Europe de l'Est, un Orient de l'intérieur. C'est ainsi que « les divisions [...] de la culture européenne », en l'occurrence, celle entre l'Europe occidentale et l'Europe orientale, deviennent « sources d'un certain exotisme », pour reprendre une formule de Jean-Marc Moura (1997 : 15). Considérer la représentation simenonienne de la Pologne permet donc d'étendre l'usage des concepts d'exotisme et d'orientalisme aux rapports interculturels intra-européens. Non seulement au niveau géographique, mais aussi symbolique, le continent européen possède bien son Occident et son Orient. 


\section{Racialisation et érotisation}

Dans les portraits physiques de ses personnages, lécrivain réactive une sorte de physiognomonie ethnique, voire raciale. Par exemple, ses Slaves se signalent par leur blondeur et la clarté de leur peau, telle, dans Stan le Tueur, cette Polonaise « aux cheveux blonds, au teint très clair, que tout le monde dans le quartier était capable de reconnaître pour une étrangère » (Simenon 2015 : 297). Le physique de l'héroïne fonctionne ici clairement comme signe de ses origines étrangères. Dans ses reportages, Simenon fait explicitement appel à des stéréotypes ethniques, p.ex. celui de "la fameuse âme slave " (Simenon 2001 : 780), sans que l’on sache précisément quel sens prêter à cette dernière qui, selon la formule de Michel Cadot, est toujours « définie en termes vagues et poétiques² " (Cadot 1973 : 96). Tout indéterminée qu'elle soit, cette mythique « âme slave » semble bien être, et pas seulement chez Simenon, l'un des éléments constitutifs d’un orientalisme intra-européen.

Dans les textes fictionnels, le narrateur simenonien est parfois ouvertement raciste, p.ex. dans ses portraits des Juifs polonais. La judéité est dans ces textes non une catégorie religieuse mais raciale. S’agissant des Juifs, c'est justement le stéréotype raciste qui prend le dessus sur la catégorisation nationale. Dans Pietr le Letton, pour construire le portrait d'Anna Gorskine, une Juive polonaise, Simenon la racialise en se servant précisément de ce genre de clichés. Elle a donc des cheveux « épais et gras comme des cheveux d'Asiatique » (Simenon 1977 : 137), cette comparaison faisant clairement référence à une particularité raciale et renvoyant à l'orientalisme. Par son apparence, la Juive polonaise se trouve ainsi associée à l'Orient asiatique, ce qui se laisse aisément expliquer car, comme le constate Ewa Maczka, « la figure de la Juive intègre pleinement l'imaginaire orientaliste » (Maczka 2010 : 81). Said a aussi relevé l'importance du mythe aryen et du racisme dans la genèse de l’orientalisme dans le cadre duquel les "Sémites " se trouvaient rangés parmi "les Orientaux inférieurs» (Said 2005 : 118). Exploitant la veine raciste dans ses aspects les plus caricaturaux, le narrateur de Pietr le Letton va jusquà prétendre que « Chaque race a son odeur, que détestent les autres races » et que, «Dans la chambre d'Anna Gorskine, elle [cette odeur] était compacte » (Simenon 1977 : 133). L’odeur « racialisée » d’Anna incommode Maigret ; la composante olfactive de son portrait est clairement péjorative.

Même si l'orientalisme est habituellement conceptualisé comme une forme de domination symbolique, il serait faux de prétendre que l'orientalisation de la Pologne soit synonyme de sa seule négativisation. Les notes positives ne sont pas entièrement absentes de l'image du pays, et surtout de ses habitants, mais elles s'inscrivent majoritairement dans un champ notionnel particulier : celui de la sensualité, voire de l'érotisme. Par ce côté aussi, la Pologne est orientale dans la mesure où, comme l'a remarqué Said, selon les discours orientalistes, « tout dans l'Orient [...] exsudait une sexualité dangereuse " (Said 2005 : 193). En accord avec cette formule ambivalente, d'une part, la réalité polonaise recèle une charge érotique fascinante, d'autre part, elle inquiète. Dans cet esprit, Simenon brosse ce portrait de Varsovie en capitale orientale :

[...] dans un grand restaurant de Varsovie, baigné de musique langoureuse, [...] des jeux de lumière augmentent la joliesse de femmes qui se savent jolies, tandis que de superbes officiers baisent les

2 L'expression de " race slave ", apparaissant sous la plume d'Edmond de Goncourt ou d'Eugène-Melchior de Vogüé que cite l'auteur, est une variante de celle d' " âme slave». 
mains comme on ne le fait plus ailleurs et [...] la nuit passe voluptueusement sans inviter personne au sommeil... (Simenon 2001 : 776).

Cette évocation des nuits de Varsovie, avec leur " musique langoureuse », leurs « superbes officiers » et leurs jolies femmes, introduit le thème traditionnel de la volupté, ou encore de la sensualité, orientales. Simenon y réactive notamment le mythe de la belle Polonaise (Malinowski 2009 : 375-388). Toute miséreuse quelle soit, la Pologne, notamment grâce à ses habitantes, exerce sur le reporter un pouvoir d'attraction. On retrouvera la même ambivalence dans les textes fictionnels de Simenon, par rapport aux personnages féminins d’origine polonaise. Ainsi, dans Pietr le Letton, Anna Gorskine, déjà citée, est-elle représentée comme à la fois repoussante et fascinante. Dès sa première apparition, est notée « une certaine beauté ", due notamment à des " yeux très sombres, à la cornée extraordinairement blanche et brillante » que le narrateur qualifie de "remarquables » (Simenon 1977 : 60). Anna est donc à la fois racialisée, comme on l'a vu, et érotisée, et ce procédé culmine au moment de son arrestation par Maigret, lorsqu’elle se trouve dénudée : "Dans la lutte, elle déchira sa robe, se trouva dépoitraillée comme à son ordinaire, magnifique d'ailleurs, avec ses yeux qui étincelaient, sa bouche tordue » (Simenon 1977 : 129). En raison de cette érotisation, l'arrestation se transforme en une scène aux connotations sexuelles fortes. C'est une véritable prise de possession - à la fois physique et visuelle - par Maigret d'une hérö̈ne auparavant présentée comme indomptable. La formule qui résume le mieux la beauté ambivalente d’Anna est sans doute celle où le narrateur constate : "Elle était belle quand même, d'une beauté vulgaire, animale » (Simenon 1977 : 142). De la conjonction des mythes de la belle Polonaise et de la belle Juive naît donc une figure animalisée. Elle s'inscrit parfaitement dans l'imaginaire orientaliste, celui de "l'Orientale sensuelle ( Tartakowsky 2012). Sexualisée et animalisée, la Polonaise simenonienne, surtout quand elle est Juive, s'avère être plus une femelle qu'une femme. Ce qui plaide en faveur de l'interprétation de ces racialisation, érotisation et animalisation comme éléments d'un imaginaire orientaliste intra-européen, c'est que d'autres femmes originaires de l'Europe de l'Est se trouvent touchées par le même phénomène. À titre d'exemple, dans Maigret et son mort, Maria, une Slovaque, est qualifiée par le narrateur de "magnifique femelle [...] d'une autre race » (Simenon 1990 : 122). La représentation des Polonaises s'intègre ainsi dans un ensemble représentationnel plus large qui a préparé l'avènement de la figure de la " fille de l'Est », très présente dans l'imaginaire occidental depuis la chute du mur de Berlin (Giabiconi 2005).

\section{Déshumanisation et animalisation}

Bien que les textes simenoniens contiennent de nombreux portraits de Polonais, cette composante anthropologique de l'image de la Pologne n'en est pas tout à fait une dans la mesure où, aux dires de Simenon, l'appartenance de certains compatriotes de Copernic à l'espèce humaine serait sujette à caution... Laltérité polonaise radicale conduit le reporter jusqu’à remettre en question l'humanité de ceux et celles qui en sont marqués, comme dans ce fragment du reportage Europe 33 : « [...] quand on contemple ce qui est entassé dans les asiles de nuit de Pologne, on hésite un bon moment à prononcer le mot homme » (Simenon 2001 : 781). Dans le cas de ces Polonais misérables, l'altérité nationale et l'altérité sociale se renforcent, créant une figure qui sert de repoussoir à une certaine 
norme humaine bourgeoise ou petite-bourgeoise qui correspond à la fois aux origines sociales de lécrivain belge $\mathrm{e}^{3}$ et aux destinataires privilégiés qu'il a inscrits dans ses textes. Dans L'Outlaw, Stanislas Sadlak, lorsqu'il est regardé par les Français, sent qu'ils le considèrent « comme un étranger, non seulement étranger au pays, mais étranger à leur espèce d'hommes » (Simenon 2010 : 29). Dans Le Fou de Bergerac, à propos de Samuel Meyer, un Juif d’origine polonaise, se trouve posée l'hypothèse selon laquelle il pourrait appartenir à « une espèce différente de l'espèce humaine ordinaire $^{4} »$ (Simenon 1975 : 95). Le Polonais de Simenon est donc susceptible de remettre en cause l'unité même de l'espèce humaine. S'il n'est pas toujours déshumanisé, il est réduit à un spécimen à part. Le discours simenonien sur les Polonais converge de la sorte avec cette «idéologie déshumanisante » qui, selon Said, « entoure l'Arabe ou le musulman » dans le discours orientaliste (Said 2005 : 41).

Cette approche « spéciste » des Polonais est encore accentuée dans ces textes où ils se trouvent explicitement animalisés, se transformant en différentes (sous-)espèces dans le bestiaire simenonien. Certains d'entre eux vivent dans l'intimité des bêtes, comme on le voit dans un extrait d'Europe $33:$ : [...] dix, quinze personnes vivent entre quatre murs, portes et fenêtres mais en hiver, en compagnie des poules, des cochons, voire de la vache» (Simenon 2001 : 778). Vivant avec les animaux, ils semblent, dans l'esprit de Simenon, s'animaliser en quelque sorte par métonymie. Dans Peuples qui ont faim, le reporter désigne les immigrants est-européens à la recherche d'une vie meilleure par l'expression « bétail humain » (Simenon 2001 : 857). Dans L'Outlaw, un Polonais blessé dans une bagarre regarde les policiers qui interviennent " comme une bête »; un petit garçon, dans la même scène, a des "yeux de chat » (Simenon 2010 : 162). Dans le même roman, les crimes prêtés à la «bande des Polonais » sont particulièrement atroces : il s'agit d'assassinats de familles entières à la hache, selon un scénario qui fait penser à De sang-froid de Truman Capote. Le commissaire Lognon les caractérise comme " des crimes si répugnants et si lâches qu'ils donnent l'impression d'avoir été commis par des bêtes féroces ", pour enfin ajouter : " Ces bêtes-là, chez nous, on les abat» (Simenon 2010 : 184). Capturée, une partie de la bande sera en effet condamnée à mort. Dans la mesure où ils auront tout d'abord été animalisés, cette condamnation émouvra sans doute peu les lecteurs et lectrices du roman. Animaliser est donc une autre manière de mettre à distance. Dans Stan le Tueur, une Polonaise criminelle est qualifiée de «femme-vampire » (Simenon 2015 : 324). La référence à cette créature mythique, mi-homme, mi-animal, caractérisée par son goût du sang, s'inscrit aussi dans le procédé de l'animalisation. La Polonaise se trouve d'ailleurs à la tête d'une bande à laquelle est attribué le même genre de crimes quà celle de L'Outlaw.

Cette «bande des Polonais » serait une bande bien réelle ayant terrorisé le Nord de la France, dans les années 1920, avant de passer en Belgique (Lemoine, Carly 2003 : 96). Son souvenir semble avoir hanté Simenon dans la mesure où elle réapparaît dans plusieurs de ses textes, parfois furtivement, comme dans le premier Maigret, Pietr le Letton, où elle est mentionnée dans cette phrase : " la fameuse bande des Polonais, dont les chefs ont fini sur l'échafaud, fut plusieurs fois mise à contribution par des malfaiteurs d'un autre rang désireux de ne pas se souiller les mains de sang " (Simenon 1977 : 88). Elle est donc censée exécuter les basses œuvres et devient synonyme de la

3 Comme le signale Danielle Bajomée, Simenon appartenait par sa naissance au milieu petit-bourgeois catholique, même s'il a essayé de s'en affranchir (Bajomée $2013: 111$ ).

4 Les deux Meyer représentés dans le roman sont des criminels : Samuel, le père, est un meurtrier sadique, le fils, vivant sous le faux nom de Rivaud, se rend coupable de parricide. 
criminalité la plus odieuse. Ses membres sont présentés comme des bêtes féroces sanguinaires, mais cette façon de les animaliser par le crime n'est qu'un avatar d'un procédé auquel sont soumis, comme nous l'avons vu, bon nombre de leurs compatriotes, selon des modalités différentes qui ont pour point commun de les déshumaniser. Cependant, à nouveau, ce ne sont pas les seuls Polonais qui se trouvent soumis à ce procédé : dans Maigret et son mort, la bande des Polonais se transforme en une bande de Tchécoslovaques à qui exactement le même type de crimes est imputé, qui sont qualifiés de " grands fauves » et opposés aux « hommes civilisés » car ils " rappellent les époques révolues de la vie dans les forêts » (Simenon 1990 : 127, 176). Dans l'univers simenonien, les Européens de l'Est sont donc des Orientaux primitivisés et animalisés face à des Occidentaux incarnant le sommet de l'évolution humaine. Leur animalisation participe de la mise en place d'une " relation hiérarchisée » que Daniel-Henri Pageaux voit comme l’un des éléments constitutifs de toute image d'une culture autre (Pageaux 1995 : 142) et qu'Edward Said tient pour l'un des traits caractéristiques de l'orientalisme dans la mesure où « l'Oriental » désigne « une espèce inférieure d’êtres humains » (Said $2005: 368$ ).

\section{Excès inquiétant}

Si des éléments plus positifs apparaissent dans l'image simenonienne de la Pologne, ils sont souvent placés sous le signe de l'excès, et donc également de la différence par rapport à une norme française, belge, ou, plus largement, occidentale. L'un des traits caractéristiques que Simenon attribue à la Pologne est cet excès en toute chose, comme on le voit dans le fragment suivant d'Europe 33 :

Je vous ai décrit la campagne, la ville, la banlieue. Il me reste à vous décrire les restaurants, les dancings, les cabarets, les cafés.

Partout, vous étiez dépaysé parce que ceétait trop pauvre. Ici, vous Parisien, vous Français, vous êtes mal à l'aise parce que c'est trop riche.

Nos cafés sont de pauvres petits cafés où il n’y a même pas d'orchestre. À Varsovie, dès onze heures du matin, on boit en musique (Simenon $2001: 780$ ).

Lon voit ici que le destinataire inscrit dans le texte est français, et plus précisément parisien, et c'est lui qui est chargé d'incarner la norme. C’est par rapport à cette dernière que la Pologne devient le pays de l'excès. Selon lécrivain belge, « c'est le plus grand des petits pays » qui " multiplie à la mesure de sa superficie les qualités et les défauts » (Simenon 2001 : 776). La Pologne serait donc en quelque sorte constitutivement excessive. Simenon prête à cet excès un effet de dépaysement. Mais l’altérité polonaise - et plus largement centre- et est-européenne - n'est pas seulement dépaysante, elle s'avère aussi inquiétante. La Pologne, mais également, plus largement, toute l'Europe médiane, cet Orient intra-européen, sont une source d'angoisse pour l'Européen de l'Ouest. Comme le formule Simenon, « [...] du nord au sud, de la Baltique à la mer Noire et à la Méditerranée, ils ont tous innocemment des allumettes plein les mains " (Simenon 2001 : 776). L'on retrouve donc à nouveau chez Simenon ce «style de pensée fondé sur la distinction [...] entre l'Orient' et [...] l'Occident'» auquel on faisait référence précédemment (Said $2005: 15$ ). 
Bien sûr, Simenon n'utilise pas les mots « Orient » et « Occident », mais son portrait de la Pologne s'inscrit dans une vision géopolitique plus globale où il oppose clairement l'Europe centrale et orientale - Pologne, Tchécoslovaquie, Lituanie, Ukraine, Roumanie, Hongrie, Yougoslavie - à celle de l'Ouest. Déjà dans Le Fou de Bergerac, le narrateur simenonien traitait en bloc «Le Centre et l'Est de l'Europe, depuis Budapest jusqu’à Odessa, depuis Tallin jusqu’à Belgrade... » (Simenon 1975 : 95), balayant d'un seul regard cette région extrêmement vaste et hétérogène, ne serait-ce que du point de vue géographique et historique. De la même manière, dans ses reportages, en bon orientaliste, Simenon réduit la diversité centre- et est-européenne à une métaphore unique. L'image des allumettes lui sert surtout à insister sur les tensions que crée le choc des nationalismes dans cette partie du continent où, à la suite de la Première Guerre mondiale et de l'effondrement des empires, de nombreuses nations ont accédé à l'indépendance et les entités étatiques se sont multipliées. À propos des Polonais, Simenon parle de leur «patriotisme agressif» (Simenon 2001 : 780) et note : "Ils l'adorent leur Pologne ! Ce n'est plus du patriotisme. C'est une religion » (Simenon 2001 : 781), pour ajouter ensuite : «Ils n'acceptent que parler Pologne, budget polonais, intégrité et grandeur polonaises» (Simenon 2001 : 782). Le " patriotisme agressif », devenu une " religion ", renvoie à une nouvelle forme d'excès, à savoir au nationalisme, compris comme un patriotisme poussé à l'extrême. Pour l'expliquer, Simenon cite ses interlocuteurs polonais qui invoquent l'époque des partages du pays entre ses voisins (de la fin du XVIII jusqu'au début du XX siècle), pour justifier leur attachement viscéral à un État nouvellement reconstitué. Même s'il les comprend dans une certaine mesure, la diffusion du nationalisme parmi les Polonais et dans les autres pays de la région lui apparaît comme un signe inquiétant, un facteur d'instabilité politique. L'excès de nationalisme peut mener au pire, pourrait-on résumer ses craintes, en paraphrasant un écrivain émigré précisément de cette partie de l'Europe qui inquiète tellement Simenon, au demeurant assez lucide sur la situation politique qui y règne dans les années 1930.

\section{Altérisation et étrangéisation linguistique}

Comme on l'a dit au début, Simenon crée une image différentialiste de la Pologne et des Polonais. Dans ses textes fictionnels, l'altérité, et parfois aussi l'étrangeté des personnages polonais, sont signalées dès le niveau onomastique. Lécrivain y hyperbolise la différence linguistique. En affublant Waldemar du patronyme de "Strvzeski », dans la nouvelle éponyme, il se livre à une fantaisie orthographique et phonétique, inspirée des sonorités du polonais. Même si on remplaçait le « $\mathrm{V}$ », très rare en polonais, par un « $\mathrm{W} »$ et le « $\mathrm{z}$ » par un « $\dot{\mathrm{Z}} »$, ce nom paraîtrait toujours peu vraisemblable du point de vue de l'onomastique polonaise. Laccumulation de consonnes crée un patronyme difficilement prononçable même pour un polonophone et, a fortiori, pour un francophone. Cette difficulté est d'ailleurs thématisée dans le texte lorsque Waldemar essaie d’apprendre au juge d'instruction Froget qui l'interroge à prononcer son nom : "Pardon... Strvzes... Vous comprenez ? Regardez ma bouche... Strvzes... comme ceci... Puis ki... Il y a peu de Français qui soient capables de prononcer mon nom correctement et c'est très désagréable " (Simenon 1978 : 91). Les difficultés de prononciation de son nom dotent le Polonais d'une identité onomastique dans laquelle son statut d'étranger est tout de suite non seulement perceptible mais aussi accentué. Si l'on examine d'autres noms " polonais » qui apparaissent dans les textes simenoniens, l'on se 
rend compte qu'il s'agit très souvent d'une onomastique fantaisiste, n'entretenant avec la langue polonaise qu'un rapport assez éloigné. Les noms que portent les Polonaises et Polonais de Simenon possèdent souvent des consonances plutôt russes ou vaguement slaves quà proprement parler polonaises. Il en va ainsi d’Anna Gorskine (Pietr le Letton), Michel Ozep, Olga Tzérewski, Stéphanie Polintskaïa (Stan le Tueur), Frida Stavitskaïa ou Yvan, censé être originaire des environs de Cracovie, ce que son prénom rend peu vraisemblable (L'Outlaw). Les noms plus vraisemblables sont rares : Stanislas Sadlak et Josef Sibirski, dans L'Outlaw, portent des prénoms qui apparaissent dans une version francisée, mais leurs noms de famille sont plus proches de lonomastique polonaise. En règle générale, l’effet mimétique n’est pas ce que semble rechercher Simenon dans sa version très personnelle de cette dernière. Les noms de ses personnages polonais fonctionnent plutôt comme des signaux conventionnels d'une altérité voire d'une étrangeté slave.

Laltérité linguistique des Polonais simenoniens est aussi produite par le fait de signaler dans la narration leurs difficultés d'élocution en français. Dans Waldemar Strvzeski, le héros éponyme, pourtant censé bien maîtriser le français, car ayant travaillé comme interprète, signale lui-même ces difficultés lorsqu'il dit : « Je ne m’inquiète pas des ragots... (C'est bien ainsi que l'on dit en français ?) ...» (Simenon 1978 : 95). Ce qui est surprenant, c’est que l'insécurité linguistique se manifeste en l'occurrence à propos d'un mot assez courant et chez un Polonais vivant et travaillant en France. Mais à un lecteur francophone de l'époque, cela ne semblait sans doute pas invraisemblable dans la mesure où, selon l'opinion courante d'alors, les Polonais ont du mal à maîtriser le français. À titre d'exemple, dans une étude sur l'intégration des immigrés polonais et italiens en Belgique, publiée en 1953, ses auteurs insistaient sur « La difficulté que les Polonais éprouvent à s'assimiler la langue... » et soulignaient que «Les difficultés de langue isolent davantage les Polonais des autochtones"(Clémens, Vosse-Smal, Minon 1953 : 363). Le texte de Simenon se fait l'écho de cette représentation des Polonais. Dans Stan le Tueur, sont mentionnés des immigrés polonais du Nord de la France qui, selon le narrateur, "pour la plupart ne parlaient pas le français » (Simenon 2015 : 290). Ce même narrateur constate aussi à propos de Michel Ozep : «Il avait un accent polonais très prononcé » (Simenon 2015 : 288). Laccent en question se manifeste notamment par une prononciation particulière du nom de Maigret : lorsque c'est Ozep qui l'articule, celui-ci est noté « Maigrette». Cette prononciation, signalée par une orthographe modifiée, devient un véritable signe particulier linguistique du personnage. Qui plus est, le narrateur insiste sur les difficultés de compréhension que crée cette idiosyncrasie phonétique : Ozep est selon lui « fatigant à écouter, d’autant plus que l’accent déform[e] les syllabes au point qu'il fa[ut] une attention soutenue pour tout comprendre » (Simenon 2015 : 295). Un blocage communicationnel potentiel guette donc à tout moment le Polonais simenonien, risquant d'entraver ses contacts avec les francophones natifs. La question de l'accent revient dans L'Outlaw où le narrateur caractérise ainsi la façon de parler de Stanislas Sadlak : "Jamais il n'avait eu un si fort accent et par surcroît certains mots ne lui venaient à l'esprit qu'en polonais ou en allemand " (Simenon 2010 : 34). En l'occurrence, une prononciation idiosyncrasique se double d'un déficit lexical. À propos d'une Polonaise dans le même texte, l'inspecteur Janvier déclare : "Elle ne sait pas un mot de français ", après quoi il pose à son compagnon cette question rhétorique : «Tu ne comprends pas le français non plus? » (Simenon 2010 : 161-162). Un accent particulier et les difficultés d'élocution deviennent des attributs récurrents de nombre de Polonais simenoniens. Ceux-ci se voient attribuer une identité linguistique très claire, dans la 
continuité de leur identité nationale d'origine, et ont du mal à accéder au statut de francophones à part entière. Une gradation s'installe entre ceux qui ignorent complètement le français et ceux qui le parlent plus ou moins bien, mais leur rapport à la langue de Simenon reste invariablement problématique.

\section{Effets (parfois pervers) de la focalisation interne}

Pierre Assouline, cité au début de cet article, évoquait, à propos des étrangers chez Simenon, la question de la compassion, autrement dit, de l'empathie. Celle-ci suppose toujours un certain degré d'identification or, nous l'avons vu, les textes simenoniens abondent plutôt en procédés de distanciation vis-à-vis de la Pologne et des Polonais. Dans les nouvelles et les romans de l'auteur belge, il existe pourtant aussi un mécanisme narratif qui pourrait favoriser l'empathie des lecteurs et lectrices pour les personnages : c'est le recours systématique à la focalisation interne et au discours indirect libre (Alavoine 2001b : 49-61). Il y a de nombreux exemples de ce procédé dans L'Outlaw où nous pénétrons, au travers de la narration, dans le psychisme de Stanislas Sadlak. Cette focalisation interne nous permet de suivre le cours de ses pensées, ce qui peut faire naître une certaine sympathie pour le héros, mais, à d'autres moments, est susceptible de renforcer l'effet de distanciation. C'est le cas lorsque nous obtenons l'accès à des pensées particulièrement choquantes, p.ex. quand le personnage forme le projet de tuer sa maîtresse qui le cache mais qui pourrait devenir un obstacle l'empêchant d'agir à sa guise ; on le voit dans ce fragment : "Si Nouchi n’avait pas été là... Ce qui serait facile, en tout cas, ce serait de se retourner, de la saisir doucement à la gorge et de serrer de toutes ses forces. Deux minutes et demie à trois minutes suffiraient...»(Simenon 2010 : 167). Ce que nous apprenons par la suite, c'est qu'il a effectivement mis à exécution ce projet de meurtre, pour que Nouchi ne l'empêche pas de cambrioler le couple Storm qui l'a recueillie. L'effet de distanciation est d'autant plus fort que le héros forme aussi d'autres projets de meurtres crapuleux : dans un premier temps, Stanislas projette en effet d'assassiner non les Storm, mais une vieille cuisinière, voisine de Nouchi, pour s'emparer de ses économies. L'usage de la focalisation interne ne produit donc pas d'effet univoque. S'il est vrai qu'il permet de suivre la naissance d'une obsession de l'argent chez le protagoniste sous l'effet d'une misère extrême - au début du roman, nous faisons sa connaissance au moment où lui et Nouchi n'ont pas de quoi manger - le fait de nous livrer les raisonnements dans lesquels il forme le projet de s'attaquer à sa compagne d'infortune ou à sa voisine âgée favorise un effet de désidentification avant même que ce projet ne soit mis à exécution. Le seul fait d'assister au déroulement des pensées du héros ne crée donc pas automatiquement d'effet d'identification ou d'empathie. Il permet certainement de mieux comprendre la psychologie du personnage et ses motivations, sans toutefois nécessairement faire de lui un être sympathique aux yeux des lecteurs et lectrices. Si nous pouvons comprendre les Polonais simenoniens, même lorsqu'ils forment des desseins criminels, il est plus difficile de s'identifier, et à plus forte raison de sympathiser, avec eux. 


\section{Conclusion}

Jean-Louis Robert prétend que, chez Simenon, « une culture autre [...] est décrite avec une évidente xénophobie » (Robert 2004 : 162). S’il y a indéniablement une composante xénophobe dans la représentation simenonienne de la Pologne et des Polonais, cette dernière ne s'y réduit pas. Incarnant souvent la négativité, y compris une négativité criminelle dans les romans policiers, les Polonais semblent aussi l'objet d'une certaine fascination de la part du romancier belge. Celle-ci est peut-être la plus sensible dans la réutilisation de la figure mythique de la belle Polonaise, à la fois repoussante et attirante du fait de son altérité qui acquiert une dimension érotique. Simenon-reporter déclare d'ailleurs explicitement à propos des Polonais : «[...] je leur voue une admiration étrange où il $\mathrm{y} a$, d'instinct, de l'affection et de létonnement, un rien de crainte aussi » (Simenon 2001 : 780). Dans cette formule, l'écrivain belge résume très bien lui-même l’ambivalence de son rapport à la Pologne et aux Polonais. Dans une certaine mesure, c'est l'une des particularités de sa façon de voir le pays qui pourrait expliquer cette ambivalence. En effet, Simenon se le représente, en accord avec une longue tradition littéraire française (Rosset 1996 : 91), comme plein de contrastes, ainsi quon le voit dans cette citation : «Vie sordide ici, vie brillante là ! Le contraste est violent !» (Simenon 2001 : 781). Or le contraste lui-même est potentiellement générateur de sentiments ambivalents. Toutefois, l'ambivalence en question tient aussi à la dimension orientaliste de la représentation, lorientalisme véhiculant toujours une part de fascination pour l'Oriental, y compris quand il est intra-européen. Quant aux aspects techniques de l'écriture simenonienne, dans les romans et les nouvelles, le recours fréquent à la focalisation interne permet de représenter les motivations des personnages, même lorsqu'ils commettent des crimes. C'est peut-être l'une des raisons principales qui font que le portrait des Polonais fictionnels est globalement plus nuancé que celui qui émerge des reportages. Dans la recension publiée à la suite de la parution du recueil Mes apprentissages, Benoît Denis se demandait justement : " pourquoi le romancier se montre-t-il plus nuancé, plus lucide et, osons le dire, plus généreux et sensible à la misère humaine que le reporter, souvent cynique et plein de ressentiment ? (Denis 2001). Cette différence tient peut-être à la spécificité des genres romanesque et celui du reportage, tels que les pratique Simenon. Son roman - et l'on pourrait dire la même chose de ses nouvelles - tel qu'il le conçoit dans la période de la maturité, continue cette tradition romanesque du XIX ${ }^{e}$ siècle qui s'est attachée à faire du personnage un agent non seulement de l'action mais aussi de la narration. Nous donnant accès à la voix et aux pensées de ses Polonais, le romancier belge semble se rapprocher d'eux, tout en nous les rendant plus proches.

En revanche, dans les reportages, ils restent des objets d'un regard invariablement distancié d'un voyageur qu'ils ne cessent d'étonner. L'image réductrice et essentiellement négative qui s'en dégage tient, pour une large part, à la conception simenonienne du reportage, exposée dans le dernier texte d'Europe 33, intitulé «Images d'Épinal ». Il y demande à ses lecteurs:

[...] à quoi se réduisent, en somme, les idées d'un peuple sur les autres peuples ? À quelques images d'Épinal qui, pour un temps, synthétisent plus ou moins bien les pays qu’elles représentent.

Ne serait-ce pas le moment de les renouveler [...] ?

Je veux en tout cas essayer, en terminant ce tour d'Europe, de crayonner quelques croquis. Les images d'Épinal ne sont jamais exactes. Mais n’est-ce pas parce qu'elles échappent à la rigueur 
mathématique qu'elles sont presque toujours plus vraies dans leur essence et dans leur symbole que la plupart des grands machins du Salon? (Simenon 2001 : 802).

Simenon s'avère être un reporter conscient de son métier et des limites de ses textes. Il ne prétend pas à l'exactitude référentielle absolue mais entend proposer à ses lecteurs des images synthétiques, simplifiées, confiant dans leurs vertus heuristiques. Il se donne aussi pour objectif de renouveler ces images, autrement dit, ses reportages doivent être par principe démystificateurs. Une part de leur noirceur foncière tient sans doute à ce principe. Simenon semble l'assumer pleinement comme partie de sa « méthode » de reporter. Reste à savoir si nous, en tant que lecteurs et lectrices, sommes prêts à accepter ce parti pris simenonien et cette image finale de la Pologne qu'il propose après avoir exposé son « discours de la méthode» :

Pour la Pologne, je propose une grande maison grise aux carreaux cassés. Dans la cave, il y a dix pauvres familles qui font chacune un enfant tous les ans. Si bien qu’on ne sait plus au juste à qui est celui qui est mort la semaine dernière.

Au rez-de-chaussée, un Juif crasseux vend des fourrures et du saucisson.

Au premier, dans un vaste appartement, un vrai noble hurle à travers la porte qu'il n'est pas là quand des créanciers se présentent.

Au second, des fonctionnaires bien habillés qui parlent français pour ne pas être compris par les domestiques.

Dans une chambre, là-haut, un officier d’artillerie qui prépare son examen... (Simenon 2001 : 803).

Voilà un portrait de la Pologne en maison délabrée, habitée par des locataires qui, sous la plume de Simenon, paraissent peu recommandables. Ce tableau synthétise en effet assez bien l'image qui ressort des reportages, avec toute sa négativité, son misérabilisme, son racisme, mais aussi quelques accents plus positifs, bien timides, il est vrai. À la fin d'Europe 33, Simenon qualifie aussi les images des différents pays qu'il y a dressées de «caricatures » (Simenon 2001 : 805). Si l'on voulait chercher une vérité dans les images simenoniennes de la Pologne et des Polonais, elle serait sans doute justement celle à laquelle peut aboutir un caricaturiste qui grossit certains traits, sans complètement évacuer la ressemblance avec le modèle.

\section{Références bibliographiques}

Alavoine, B. (2001a). Les voyages de Georges Simenon. Du récit-reportage à la fiction. In P. Antoine, \& M.-Ch. Gomez-Géraud (Éds.), Roman et récit de voyage (pp. 175-182). Paris : Presses de l'Université de Paris-Sorbonne.

. (2001b). Qui raconte chez Simenon? Originalité du jeu avec la voix et le point de vue. Cahiers de narratologie, 10, 49-61.

Assouline, P. (2017). La vision de l'étranger de Simenon est très intéressante, il a une vraie compassion. $<$ https://www.franceculture.fr/emissions/les-nuits-de-france-culture/pierre-assouline-simenon-est-nesous-le-signe-de-lexces-1>. 
Bajomée, D. (2013). Badinages, étreintes et soupirs. Simenon, ce polisson. Les Cahiers de l'Herne. Georges Simenon, 110-118.

Bordas, É. (2002). Image, imagologie. In P. Aron, D. Saint-Jacques, \& A. Viala (Éds.), Le Dictionnaire du littéraire (pp. 288-289). Paris : PUF.

Cadot, M. (1973). Naissance et développement d'un mythe ou l'Occident en quête de lâme slave. Revue des Études Slaves, 49, 91-101.

Clémens, R. ; Vosse-Smal, G. ; \& Minon, P. (1953). L’Assimilation culturelle des immigrants en Belgique. Italiens et Polonais dans la région liégeoise. Liège : Imprimerie H. Vaillant-Carmanne.

Denis, B. (2001). Simenon (Georges), Mes Apprentissages. Reportages 1931-1946. Textyles, 20. <http://journals.openedition.org/textyles/986>.

Eskin, S. (1990). Simenon. Une biographie. Paris : Presses de la Cité.

Giabiconi, D. (2005). Filles de l'Est : construction d'une féminité racisée. Séminaire EFFIGIES du 14 mai 2005. <https://halshs.archives-ouvertes.fr/halshs-00003981/document>.

Halen, P. (1997). Propositions sur l'exotisme, avec une esquisse de Simenon en écrivain colonial. Traces, 9, 193-208.

Le Fou de Bergerac. (s.d.). <https://www.toutsimenon.com/oeuvre/tout-simenon/fiche-livre/17-Le-fou-deBergerac_3915>.

Lemoine M. ; \& Carly M. (2003). Les Chemins belges de Simenon. Liège : Éd. du CEFAL.

Maczka, E. (2010). La « belle Juive », avatars d'une figure de l'Autre en littérature française. Scripta Judaica Cracoviensia, 8, 77-92.

Malinowski, W. M. (2009). La belle Polonaise dans la littérature française : pour une histoire du stéréotype. Kwartalnik neofilologiczny, LVI, 4, 375-388.

Moura, J.-M. (1997). L'exotisme, une notion aux significations voyageuses. Traces, 9, 9-20.

Pageaux, D.-H. (1995). Recherche sur l'imagologie : de l'histoire culturelle à la poétique. Revista de Filología Francesa, 8, 135-160.

. (2007). Littératures et cultures en dialogue. Paris : L'Harmattan.

Pantkowska, A. (2006). Le stéréotype de la Pologne et des Polonais dans les lettres belges de langue française. In M. Forycki, \& M. Serwański (Éds.), Amis et ennemis héréditaires. Les Stéréotypes nationaux (pp. 117-129). Poznań : Instytut Historii UAM.

Pietr le Letton. (s.d.). <https://www.toutsimenon.com/oeuvre/tout-simenon/fiche-livre/17-Pietr-le-Letton_4012>.

Reportage. (s.d.). In Le Trésor de la langue française informatisé. <http://stella.atilf.fr/Dendien/scripts/tlfiv5/ visusel.exe?13;s=768756915;r=1;nat=;sol=4; $>$.

Robert, J.-L. (2004). Maigret à Paris. Sociétés et représentations, 17, 159-169.

Said, E. (2005). L'Orientalisme. L'Orient créé par l'Occident. Trad. C. Malamoud. Paris : Seuil.

Sensationnel. (s.d.). In Le Trésor de la langue française informatisé. <http://stella.atilf.fr/Dendien/scripts/tlfiv5/visusel.exe?12;s=1470642630;r=1;nat=;sol=1;>.

Simenon, G. (1975). Le Fou de Bergerac. Paris : Le livre de poche.

. (1977). Pietr le Letton. Paris : Presses Pocket.

. (1978). Waldemar Strvzeski. In Idem, Les 13 coupables (pp. 90-103). Paris : Presses Pocket.

. (1990). Maigret et son mort. Paris : Presses de la Cité.

. (2001). Mes apprentissages. Reportages 1931-1946. Paris : Omnibus.

(2010). L'Outlaw. Paris : Gallimard. 
. (2015). Stan le Tueur. In Idem, Les Nouvelles Enquêtes de Maigret (pp. 285-326). Paris : Gallimard.

Tartakowsky, E. (2012, 5 juillet). « La belle Juive» : l'imaginaire oriental au féminin. La Vie des idées, <https:// laviedesidees.fr/La-belle-Juive-l-imaginaire-oriental-au-feminin.html>.

Waldemar Strvzeski. (s.d.). <https://www.toutsimenon.com/oeuvre/tout-simenon/fiche-livre/17-Waldemar-Strvzeski_4126>. 